\title{
Importancia de la cardiooncología. Cómo detectar la insuficiencia cardiaca subclínica
}

\author{
Importance of cardio-oncology. How to detect suclinical heart failure \\ Jesús De Rubens-Figueroa ${ }^{1 *}$ y Rocío Cárdenas-Cardós ${ }^{2}$ \\ ${ }^{1}$ Servicio de Cardiología, Laboratorio de Ecocardiografía; ${ }^{2}$ Servicio de Oncología. Instituto Nacional de Pediatría, Ciudad de México, México
}

\begin{abstract}
Resumen
Hoy una de las principales causas de mortalidad es el cáncer. La supervivencia en pacientes con cáncer ha incrementado de 1970 (25\%) a la actualidad (80\%). A raíz de la introducción de las antraciclinas como tratamiento de cáncer desde 19601970, más del 60\% de los pacientes son tratados con estos agentes; sin embargo, la exposición de quimioterápicos conlleva las enfermedades cardiovasculares como la principal causa de mortalidad en enfermos supervivientes de cáncer en el s. XXI. Hay múltiples factores que incrementan la sensibilidad de cardiotoxicidad inducida por antracíclicos. En 1970 el estándar de oro para la detección de disfunción ventricular era la biopsia endomiocárdica, en forma posterior la detección y manejo de la cardiotoxicidad fue guiada por los síntomas, en 1981 la detección de cardiotoxicidad fue reportada con la determinación de la fracción de expulsión del ventrículo izquierdo (FEVI) por ecocardiografía 2D. En la actualidad el ecocardiograma 3D para FEVI y volúmenes sistólico y diastólico han presentado una alta correlación de los valores obtenidos por resonancia magnética para la evaluación de la función cardiaca. Hoy en día la ecocardiografía strain, strain-rate y speckle tracking se utilizan para determinar la función miocárdica regional y global. Para una valoración integral estos resultados se pueden complementar con biomarcadores cardiacos (troponinas y propéptido natriurético tipo B) y cambios electrocardiográficos. De esta forma se puede detectar insuficiencia cardiaca subclínica y dar un tratamiento oportuno.
\end{abstract}

Palabras clave: Cardiotoxicidad. Cáncer. Strain. Insuficiencia cardiaca. Quimioterapia.

\begin{abstract}
Today one of the main causes of mortality is cancer. Survival in cancer patients has increased from 1970 (25\%) to the present (80\%). Following the introduction of anthracyclines as a cancer treatment since 1960-70, more than 60\% of patients are treated with these agents, although chemotherapeutic exposure leads to cardiovascular diseases as the main cause of mortality in surviving patients. of cancer in the $21^{\text {st }}$ Century. There are multiple factors that increase the sensitivity of anthracyclic-induced cardiotoxicity. In 1970 the gold standard for the detection of ventricular dysfunction was endomyocardial biopsy, subsequently the detection and management of cardiotoxicity was guided by symptoms, in 1981 the detection of cardiotoxicity was reported with the determination of the ejection fraction of the left ventricle (LVEF), by $2 D$ echocardiography. Currently, the 3D echocardiogram for LVEF and systolic and diastolic volumes have presented a high correlation of the values obtained by magnetic resonance imaging for the evaluation of cardiac function. Today strain, strain-rate and speckle tracking echocardiography are used to determine regional and global myocardial function. For a comprehensive assessment, these results can be complemented with cardiac biomarkers (troponins) and electrocardiographic changes. In this way, subclinical heart failure can be detected and timely treatment can be given.
\end{abstract}

Key words: Cardiotoxicity. Cáncer. Strain. Heart failure. Chemotherapy.

\footnotetext{
Correspondencia:

Fecha de recepción: 11-12-2019

Fecha de aceptación: 07-02-2021

*Jesús De Rubens-Figueroa

E-mail: derubens@ @otmail.com

DOI: $10.24875 /$ ACM.19000394

1405-9940 / @ 2020 Instituto Nacional de Cardiología Ignacio Chávez. Publicado por Permanyer. Este es un artículo open access bajo la licencia

CC BY-NC-ND (http://creativecommons.org/licenses/by-nc-nd/4.0/).

Disponible en internet: 12-04-2021 Arch Cardiol Mex. 2021;91(2):229-234 www.archivoscardiologia.com
} 


\section{Introducción}

Hoy una de las principales causas de mortalidad es el cáncer. La supervivencia en pacientes con cáncer ha incrementado de 1970 (25\%) a la actualidad (80\%), debido a la prevención, diagnóstico temprano y tratamiento oportuno'; sin embargo, el tratamiento oncológico conlleva complicaciones a mediano y largo plazo, la principal es la enfermedad cardiovascular prematura, debido a la exposición a quimioterápicos como las antraciclinas ${ }^{2}$. Por lo tanto, las enfermedades cardiovasculares son la mayor causa de mortalidad en enfermos supervivientes de cáncer ${ }^{3}$. La mortalidad por afección cardiaca es 3.4 veces mayor que la esperada en la población general ${ }^{4}$.

A raíz de la introducción de las antraciclinas como tratamiento de cáncer desde 1960-1970, más del 60\% de los pacientes con cáncer son tratados con estos agentes; sin embargo, muchos de ellos se han asociado con disfunción ventricular, cardiomiopatía, prolongación del QT, insuficiencia valvular, isquemia miocárdica, pericarditis, arritmia, síncope, coronariopatía y muerte súbita ${ }^{1,5-7}$.

La afectación del miocardio respecto al tiempo de inicio del tratamiento con antraciclinas se ha dividido en: aguda (primera semana de administración), temprana o subaguda (de una semana al primer año) y crónica (mayor a un año de administración), e incluso se ha detectado un $10 \%$ de los pacientes con insuficiencia cardiaca hasta 20 años después de haber iniciado el tratamiento ${ }^{8-10}$.

Queremos mencionar la importancia de detectar los factores de riesgo, como: algunos polimorfismos, obesidad, hipertensión arterial sistémica, edad menor a cuatro años, sexo femenino, síndrome metabólico, radioterapia mayor de $5 \mathrm{~Gy}$, anticuerpos monoclonales, trasplante de células hematopoyéticas, mitoxantrona, 5-fluoracilo, agentes alquilantes como ciclofosfamida, cisplatino, agentes bilógicos como trastuzumab y rituximab, inhibidores de tirosinacinasa como imitanib, sunitinib y dazatinib ${ }^{8-11}$. El de mayor riesgo en los análisis estadísticos es la dosis acumulada $\geq 300$ $\mathrm{mg} / \mathrm{m}^{2}$ de antraciclinas, con una incidencia hasta del $14.6 \%$.

El mecanismo de cardiotoxicidad por los antracíclicos es la liberación de radicales libres y peróxidos que dañan la fibra cardiaca, inhiben la síntesis de proteínas, con inducción de la cascada de apoptosis y muerte celular $^{10,12}$. Las antraciclinas inhiben la síntesis de ADN y ARN, ocasionando ruptura de filamento con efecto mutágeno y carcinógeno, formación y depósito de hierro férrico en los cardiomiocitos, disminuyendo el grupo quinona, formando radicales superóxidos y peróxido de hidrógeno, que lesionan la integridad de la membrana mitocondrial, inhiben la entrada de iones de calcio y sodio intracelular, necrosis y apoptosis, disminuyendo la contractilidad miocárdica, ocasionando fibrosis cardiaca, insuficiencia cardiaca y cardiomiopatía dilatada $y / 0$ restrictiva ${ }^{13-17}$. Por otro lado, incrementan las citocinas proinflamatorias induciendo la liberación de histamina y factor de necrosis tumoral alfa e interleucina 2, provocando miocardiopatía dilatada y disfunción betaadrenérgica ${ }^{15}$.

El primer reporte de cardiotoxicidad se realizó en Nueva York en $1967^{18}$. En 1970 el estándar de oro para la detección de disfunción ventricular era la biopsia endomiocárdica, hoy en día se ha desechado esta posibilidad invasiva, sobre todo en niños, gracias a los avances tecnológicos en los métodos de detección de cardiotoxicidad $^{10}$.

En 1980 la detección y manejo de la cardiotoxicidad fue guiada por los síntomas y con dificultad, ya que hay síntomas que son similares para la insuficiencia cardiaca y para el cáncer, como la disnea, la fatiga, el edema periférico, etc. En 1981 la detección de cardiotoxicidad fue reportada con la determinación de la fracción de expulsión del ventrículo izquierdo (FEVI) por ecocardiografía 2D. En el año 2000 se reportaron limitaciones en la FEVI, como un marcador imperfecto, porque es insensible a los cambios tempranos de la disfunción cardiaca, con conocidas limitaciones, donde el deterioro es en los estados tardíos de la enfermedad ${ }^{19,20}$.

El ecocardiograma tridimensional (3D) ha presentado una alta correlación para la evaluación de la función cardiaca de la FEVI y los volúmenes sistólico y diastólico con los valores obtenidos por resonancia magnética (RM) (r: 0.96, r: 0.97 y r: 0.93, respectivamente). En cuanto a la FEVI, se considera anormal cuando está por debajo del $5 \%$ de la cifra normal del $53 \%$ o un $10 \%$ por debajo de la FEVI inicial en pacientes asintomáticos ${ }^{21,22}$.

Hay estudios actuales donde se ha estudiado adecuadamente la afección en el ventrículo izquierdo, sin embargo, pocos estudios muestran que las antraciclinas pueden también afectar la función ventricular derecha, un concepto que no ha sido adecuadamente explorado aún ${ }^{23}$.

Hoy en día los avances en imagen cardiaca, especialmente en los no invasivos, son de gran utilidad, como la ecocardiografía strain (deformación miocárdica) y el strain-rate (velocidad de deformación). La 
medición de ambas para la función miocárdica regional y global derivadas del uso de strain 2D es el speckle tracking. Estas técnicas ecocardiográficas miden la deformación y desplazamiento existente en la contracción cardiaca, la cual tiene varios tipos o direcciones: Iongitudinal (base-ápex), circunferencial y radial. También hay que tomar en cuenta que las fibras miocárdicas también tienen mecanismos de torsión y rotación ${ }^{8,24,25}$, que se estudiarán con más detalles en los próximos años.

La imagen de strain (deformación miocárdica)/strain-rate (velocidad de acortamiento) es la medición de las velocidades del movimiento de la fibra miocárdi$\mathrm{ca}$, en su desplazamiento en movimientos de traslación/rotación, medido en gradiente en direcciones radial/longitudinal y la cual puede ser medida en diferentes segmentos miocárdicos. Este es un método muy útil para reconocer la deficiencia en la deformación y acortamiento de la fibra miocárdica antes de su manifestación clínica como insuficiencia cardiaca $^{26}$.

El strain es el porcentaje de cambio de tamaño de la fibra corregida por el tamaño original que se manifiesta en la siguiente fórmula: Strain $=(L 1-L 0 / L 0$, donde L1 es igual a la longitud de la fibra en su máxima contracción y $L 0$ es la longitud en reposo. El valor de strain se expresa en porcentaje de cambio de tamaño y puede ser positivo o negativo dependiendo de si el desplazamiento se acerca o se aleja del punto de referencia ${ }^{26}$.

Por otro lado, el valor de strain-rate se obtiene del conocimiento de la longitud basal de una fibra (L0) $y$ las velocidades iniciales y final (V0, V1) a los cuales se deforma la fibra. Se puede calcular el cambio en velocidad entre los dos puntos y corregirlo, por la longitud de la fibra con la siguiente fórmula: (V0-V1)/L026.

Para medir el strain y strain-rate cardiaco, que evalúa el desplazamiento de la fibra miocárdica con la técnica de speckle-tracking a lo largo del ciclo cardiaco puede ser por Doppler de tejidos o por rastreo de moteado con ecocardiograma 2D y así detectar diferentes segmentos miocárdicos (speckle tracking) con diferentes características de profundidad (frame-rate) (40-90 frames/s o $\geq 40 \%$ de la función cardiaca) en los diferentes segmentos estudiados, durante ese ciclo cardiaco, ya sea en sístole como en diástole y de manera simultánea se informará el strain global de los 18 segmentos cardiacos $^{22}$.

La disminución del strain longitudinal y o radial en pacientes sometidos a quimioterapia son indicadores en forma temprana y de alta sensibilidad de toxicidad miocárdica por antraciclinas.

En cuanto a la función diastólica, se ha reportado que los parámetros ecocardiográficos (E/A transmitral, prolongación de los tiempos de relajación isovolumétrica) preceden a disminuciones significativas de la $\mathrm{FEVI}$, lo que parece corresponder a un signo temprano de disfunción del ventrículo izquierdo en pacientes tratados con quimioterapia ${ }^{22}$.

En forma general se consideran parámetros normales del strain global longitudinal (SGL) en niños de $-19.4(-20.4$ a $-18.9 \%)$ y en niñas de $-20.5 \pm 2.2^{22}$; entre -16 y $-18 \%$ son intermedios $y<16 \%$ anormales $^{19,23}$ (Tabla 1). Es de importancia mencionar que se recomienda tener determinaciones ecocardiográficas basales (previos a la quimioterapia), tomados de referencias de valores $Z$ para los niños en el strain del ventrículo izquierdo, que nos servirán para comparación con las medidas posteriores de control en forma personalizada, ya que no hay valores precisos normales universales para niños ${ }^{20,27}$. El seguimiento por ecocardiografía es variable según cada centro hospitalario, pero en consenso se recomienda un primer control al mes del inicio de la quimioterapia, tres, seis y doce meses para la valoración temprana; en casos especiales se puede adelantar la valoración ${ }^{8}$.

En un estudio realizado en 2012 se detectó que en pacientes con administración de antracíclicos el SGL se alteró en el primer control ecocardiográfico, en cambio la FEVI disminuyó en forma más tardía al control de los ocho meses ${ }^{28,29}$ (Fig. 1). También es muy importante determinar la disfunción diastólica, ya que es la primera manifestación de cardiotoxicidad por antraciclinas ${ }^{30}$.

Para una valoración integral estos resultados se pueden complementar con biomarcadores cardiacos (se dice que las troponinas detectan la lesión y muerte de los cardiomiocitos y propéptido natriurético tipo B), los cuales se recomiendan tomar en una determinación basal y en forma posterior durante los controles, para realizar una comparación con los resultados anteriores, esto nos proporciona datos de inflamación que nos son de gran utilidad en la valoración del paciente, que junto a los resultados ecocardiográficos nos da una idea cercana del daño miocárdico.

También nos es útil el electrocardiograma para vigilar arritmias ocasionadas por la administración de antraciclinas como: QT largo, taquicardia ventricular, supraventricular y bloqueo auriculoventricular completo ${ }^{10,16}$. 
Tabla 1. Cifras de control normales

\begin{tabular}{|l|c|c|c|}
\hline Edad en años & Strain global longitudinal & Strain global circunferencial & Strain global radial \\
\hline $1-4$ & $-20.7 \pm 1.30$ & $-20.0 \pm 0.34$ & \\
\hline $5-9$ & $-21.0 \pm 1.30$ & $-20.9 \pm 2.00$ \\
\hline $10-14$ & $-21.8 \pm 1.30$ & $-21.5 \pm 1.70$ & $38.6 \pm 14.2$ \\
\hline $15-19$ & $-22.5 \pm 1.30$ & $-21.9 \pm 2.10$ & \\
\hline
\end{tabular}

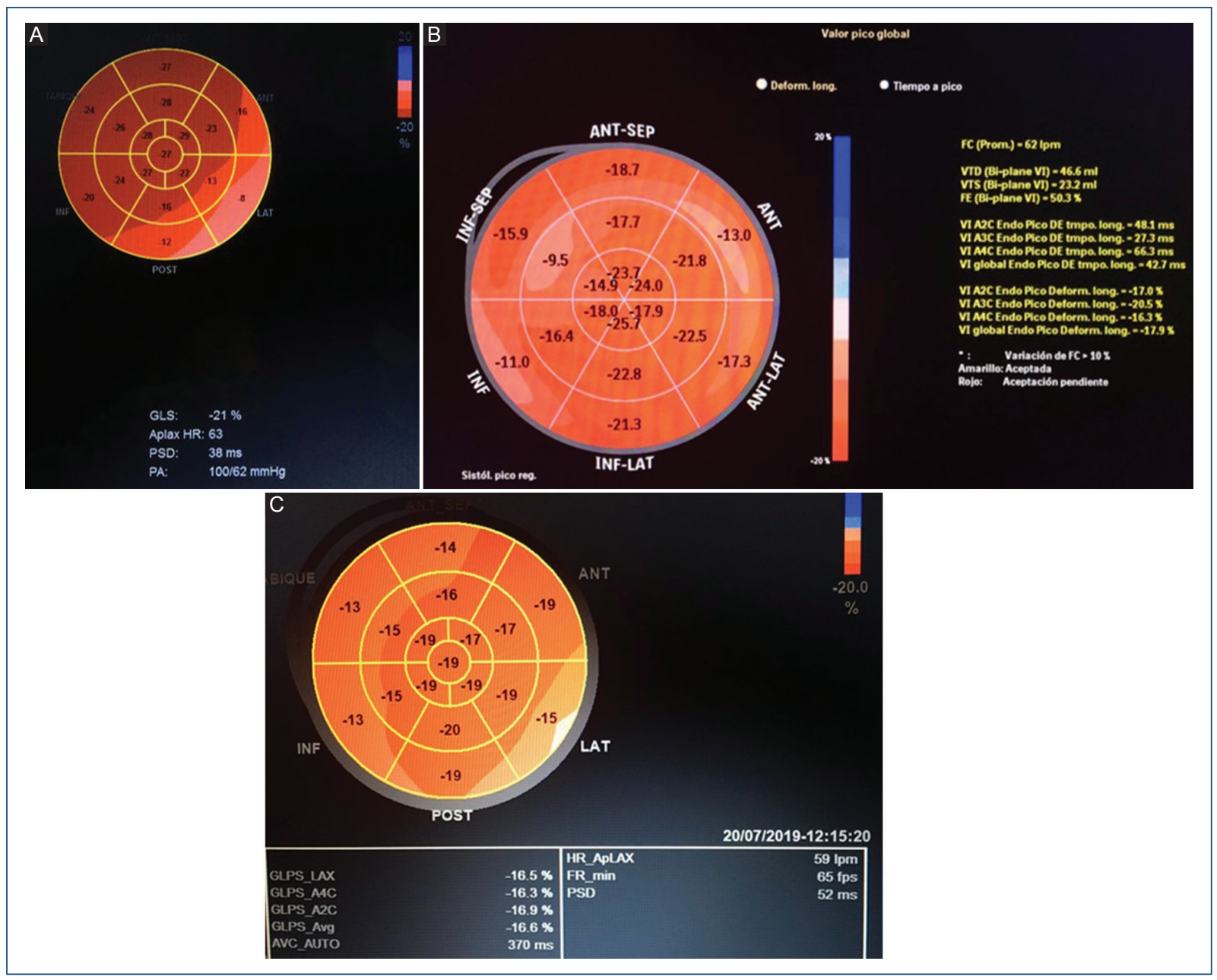

Figura 1. Donde se observa tres momentos de la evolución en el control strain de la fibra miocárdica en un paciente con administración de antraciclinas. A: medida basal (previo al tratamiento): FE 60\% con SGL $-21.0 \%$. B: seis meses después: FE $50.3 \%$ con SGL -17.9\%. C: doce meses después con FE 49\% con SGL $-16.6 \%$.

FE: fracción de eyección; SGL: strain global longitudinal.

En cuanto a los parámetros de strain, se sugirió que la reducción del SGL > 15\% con el parámetro basal del $100 \%$, tiene un importancia subclínica, así como disminución del 10\% de la fracción de eyección basal10,29 (Fig. 1).

El corazón tiene una reserva significativa y el daño que altera los parámetros a la función sistólica y diastólica se llega a manifestar hasta que la reserva cardiaca se ha perdido, así es que el daño cardiaco puede no llegar a presentarse hasta años o décadas después del tratamiento cardiotóxico y la disfunción ventricular tiene una media de supervivencia de 7.1 años ${ }^{4,22,31}$. 
Probablemente el seguimiento a largo plazo se deba hacer a los 2, 5 y 10 años posteriores a la quimioterapia ${ }^{32}$.

En cuanto al diagnóstico, la resonancia magnética nos es útil para valorar la función ventricular y se recomienda cuando hay pobre calidad de la imagen del ecocardiograma, o cuando se necesiten precisar algunos otros datos y/o bordes endocárdicos.

Para reducir la cardiotoxicidad que produce daño subclínico, tenemos tres opciones: a) disminuir la concentración de antracíclicos (no mayor a $300 \mathrm{mg} /$ $\mathrm{m}^{2}$ acumulado); b) desarrollar análogos menos cardiotóxicos (antraciclinas liposomales, daunorubicina), y c) administración de agentes cardioprotectores para prevenir la remodelación miocárdica y la progresión a la insuficiencia cardiaca como dexrazoxano (quelante de hierro y estimulante de radicales libres de oxígeno). Y para el tratamiento contamos con diuréticos, inhibidores de la enzima convertidora de angiotensina como el enalapril, y betabloqueadores como carvedilol, estatinas, metoprolol e bisoprolol, vitaminas $A, E, C$, coenzima $Q$ y carnitina ${ }^{16,22,33,34}$. Estas opciones pueden recuperar más del $50 \%$ de la $\mathrm{FEVI}$, con lo que sobreviven el $85 \%$ de los pacientes, aumentando la frecuencia cardiaca.

A últimas fechas se ha mencionado como profiláctico los anticuerpos contra el gen HER2/Neu (ErbB2), disminuyendo el riesgo de recurrencia y muerte en los pacientes gen HER2 positivos ${ }^{22}$.

La miocardiopatía inducida por antraciclinas se ha asociado a un pronóstico especialmente desfavorable, con una mortalidad a dos años de hasta el $60 \%$.

La mortalidad por cardiotoxicidad hoy en día para algunas revisiones es del $10 \%$, pero aumenta en forma notable con dosis acumuladas de antraciclinas ${ }^{29,34}$.

\section{Conclusiones}

Para disminuir la morbimortalidad de los pacientes con cáncer tratados con quimioterápicos es necesario detectar en forma temprana y subclínica la disfunción ventricular con ecocardiografía strain. Por lo tanto, a todo paciente que recibe quimioterápicos potenciales a cardiotoxicidad deberá de darse seguimiento clínico sistemático, con biomarcadores y ecocardiográfico, para dar diferentes opciones de tratamiento que recuperen más del $50 \%$ la función ventricular y de esa forma evitar la insuficiencia cardiaca.

\section{Financiamiento}

La presente investigación no ha recibido ayudas específicas provenientes de agencias del sector público, sector comercial o entidades sin ánimo de lucro.

\section{Conflicto de intereses}

Los autores declaran no tener conflicto de
intereses.

\section{Responsabilidades éticas}

Protección de personas y animales. Los autores declaran que para esta investigación no se han realizado experimentos en seres humanos ni en animales.

Confidencialidad de los datos. Los autores declaran que han seguido los protocolos de su centro de trabajo sobre la publicación de datos de pacientes.

Derecho a la privacidad y consentimiento informado. Los autores declaran que en este artículo no aparecen datos de pacientes.

\section{Bibliografía}

1. Shankar SM, Marina N, Hudson MM, Hogdson DC, Adams J, Landier W, et al. Monitoring for cardiovascular disease in surviviors of childhood cancer: report from the cardiovascular disease Task Force of the children's oncology group. Pediatrics. 2008;121:387-96.

2. Rathe M, Torp C NL, Oxhoj H, Nielsen G. Long-term cardiac follow-up of children treated with anthracycline doses of $300 \mathrm{mg} / \mathrm{m}^{2}$ or less for acute lymphoblastic leukemia. Pediatr Blood Cancer. 2010;54:444-8.

3. Kucharska W, Negrusz-Kawecha M, Gromkowska M. Cardiotoxicity of oncological treatment in children. Adv Clin Exp Med. 2012;21:281-8.

4. Fidler MM, Reulen RC, Henson K, Kelly J, Cutter D, Levit GA, et al. Population-based long-term cardiac-specific mortality among $34489 \mathrm{fi}$ ve-year survivors of childhood cancer in Great Britain. Circulation. 2017;135:951-63.

5. Kremer LC, Caron HN. Anthracycline carwdiotoxicity in children. N Engl J Med. 2004;351:120-1.

6. Godishala A, Young S, Asnani A. Cardioprotection in the modern era of cancer chemotherapy. Cardiol Rev. 2018;26:113-21.

7. Armenian SH, Armstrong GT, Avne G, Chow EJ, Erhardt MJ, Ky B, et al. Cardiovascular disease in surviviors of childhood cancer. Insights into epidemiology, pathophysiology and prevention. J Clin Oncol. 2018:36:2135-44.

8. Plana JC, Galderisi M, Barac A, Ewer MS, Ky B, Scherrer CM. et al. Expert consensus for multimodality imaging evaluation of adult patients during and after cancer therapy: A report from the American Society of Echocardiography and the European Association of cardiovascular imaging. J Am Soc Echocardiogr. 2014;27:911-39.

9. González OA, Machín GS, Arencibia NA, Collazo AJ, Rivera KC Bravo POL, et al. Evaluación ecocardiográfica longitudinal en pacientes con leucemia linfoide aguda que recibieron antracilinas durante la edad pediátrica. Rev Cub Hematol, Inmunol y Hemoter. 2016;32:470-82.

10. Asnani A, Neilan GT, Tripathy D, Scherrer CM. Clinical manifestations, monitoring and diagnosis of anthracycline-induce cardiotoxicity. Topic 2018.

11. Bini I, Asaftei DS, Riggi Ch, Tirtei E, Manicone R, Biasin E, et al. Anthracycline-induced cardiotoxicity in patients with paediatric bone sarcoma and soft tissue sarcoma. Cardiol Young. 2017;27(9):1815-22.

12. Cardenas CR. Mecanismos de cardiotoxicidad de la quimioterapia. Gac Mex Oncol. 2009;8:3-7.

13. Navarrete REM, Zapata T MM, Vizcaíno AA, Garduño EJ, Dorantes AE, Palomo C MA. El ecocardiograma y su papel en niños con cáncer. Bol Med Hosp Infant Mex. 2013;70:133-7.

14. Larussi D, Indolfi P, Casale F, Martino V, Di Tullio MJ, Calabró R. Anthracycline-induced cardiotoxicity in children with cancer: Strategies for prevention and management. Paediatr Drugs. 2005;7:67-76. 
15. Hurtado NS, Mejía CAM, Sanabria CHA. Cardiotoxicidad por quimioterapia. Un enfoque práctico para el clínico. Insuficiencia Cardiaca. 2011;3:131-43.

16. Lipshultz SE, Adams MJ, Colan SD, Constine LS, Herman EH, Hsu DT, et al. Long term cardiovascular toxicity in children, adolescents and Young adults who receive cancer therapy pathophysiology, course, monitoring, management, prevention and research directions: a scientific statement from the American Heart Association. Circulation. 2013;128:1927-95.

17. Van der Pal JH, Van Dijk, Geskus BR, Kok EW, Koolen M, Sieswerda E et al. Valvular abnormalities detected by echocardiography in 5-year survivors of childhood cancer: a long-term follow-up study. Int J Radiation Oncol Biol Phys. 2015;91:213-22.

18. Tran CJ, Ruble K, Loeb MD, Chen RA, Thompson RW. Automated functional imaging by $2 \mathrm{D}$ speckle tracking Echocardiography reveals high incidence of abnormal longitudinal strain in a cohort of pediatric oncology patients. Pediatr Blood Cancer. 2016;63:1075-80.

19. Negishi T, Negishi K. Echocardiographic evaluation of cardiac function after cancer chemotherapy. J Echocardiogr. 2018;16:20-7.

20. Pignatelli RH, Ghazi P, Chandra B RS, Thompson P, Cui Q, Castro J. Abnormal myocardial strain indices in children receiving anthracycline chemotherapy. Pediatr Cardiol. 2015;36:1610-6.

21. Narayan V, Ky B. Common cardiovascular complications of cancer therapy: Epidemiology, risk prediction, and prevention. Annu Rev Med. 2018:69:97-111.

22. Plana JC. Chemotherapy and the heart. Rev Esp Cardiol. 2011;64: 409-15.

23. Thavendiranathan P, Poulin F, Lim KD. Use of myocardial strain imaging by echocardiography for the early detection of cardiotoxicity in patients during and after cancer chemotherapy: a systematic review. J Am Coll Cardiol. 2014;63:2751-68

24. Spewak BM, Williamson SR, Mertens CA, Border LW, Meacham RL, Wasilewski-Masker JK. Yield of screening echocardiograms during pediatric follow-up in survivors treated with anthracyclines and cardiotoxic radiation. Pediatr Blood Cancer. 2017;64(6).
25. Olaya $\mathrm{P}$, Sanchez J, Osorio LF. Strain y strain rate para dummies. Rev Colomb Cardiol. 2011;18:340-4

26. Ganame J, Mertens L. Quantitative methods in echocardiography-advanced techniques in the assessment of ventricular function. En: Eidem WB, Cetta F, O'Leary WP, editores. Echocardiography in pediatric and adult congenital heart disease. Filadelfia: Lipincott Williams \& Wilkinson; 2010. pp. 57-61.

27. Marcus KA, Mavinkurve-Groothuis AMC, Barends M, van Dijk A, Feuth T, de Korte $C$, et al. Reference values for myocardial two-dimensional strain echocardiography in a healthy pediatric and young adult cohort. $\mathrm{J}$ Am Soc Echocardiogr. 2011;24:625-36.

28. Poterucha JT, Kutty S, Lindquist RK, Li L, Eidem BW. Changes in left ventricular longitudinal strain with anthracycline chemotherapy in adolescents precede subsequent deceased left ventricular ejection fraction. J Am Soc Echocardiogr. 2012;25:733-40.

29. Cilsal E, Oguz DA, Tunaoglu SF, Kula S, Pektas A. Subclinical reduction in left ventricular funciton using triplane and 2D speckle tracking echocardiography aftr anthracycline exposure in children. Anatol $\mathrm{J} \mathrm{Cardiol}$ 2018;19:58-66.

30. Calabrese V, Menna P, Annibali O, Armento G, Carpino A, Cerchiara E, et al. Early diastolic dysfunction after cancer chemoterapy: Primary endpoint results of a multicenter cardio-oncology study. Chemoterapy. 2018;63:55-63.

31. Wang TJ, Evans JC, Benjamin EJ, Levy D, Le Roy EC, Vasan RS. Natural history of asymptomatic left ventricular systolic dysfunction in the community. Circulation. 2003;108:977-82.

32. López FT, García MA, Beltrán SA, Luis MA, Sanz GR, Ramos MP, et al. Cardio-Onco-Hematología en la práctica clínica. Documento de consenso y recomendaciones. Rev Esp Cardiol. 2017;70:474-86.

33. Loar RW, Noel CV, Tunuguntka H, Colquitt JL, Pignatelli RH. State of the art review: Chemotherapy-induced cardiotoxicity in children. Cong Heart Dis. 2018;13:5-15.

34. Navarrete HS, Castellanos MA, Chaparro SA. Cardiotoxicidad por quimioterápicos. Un enfoque práctico para el clínico. Insuficiencia Cardiaca. 2011;6:131-43. 